\title{
Improving Reliability of a fire-fighting pump set with Axiomatic Design
}

\author{
Gabriele Arcidiacono ${ }^{1}$, Pierpaolo Placidoli ${ }^{1}{ }^{\star}$, Stefano Nuzzi ${ }^{2}$, and Marco Molon ${ }^{3}$ \\ ${ }^{1}$ Department of Innovation \& Information Engineering, G. Marconi University, Via Plinio 44, 00193 Rome, Italy \\ ${ }^{2}$ Lean Six Sigma Researcher, Leanprove, Via A. La Marmora 45, 50121 Firenze, Italy \\ ${ }^{3} R \& D$ Department DAB PUMPS Spa, Via Marco Polo 14, 35035 Mestrino (PD), Italy
}

\begin{abstract}
This paper introduces a case study featuring Axiomatic Design and Multi-Level Hierarchical model (MLH) applied to redesign a fire-fighting pump set. In particular, two different design concepts are presented to be applied to the supporting frame of the system to limit a vibration problem that can arise during potential malfunctioning of the fire-fighting pump. The selection of the best design has been carried out through reliability evaluation process and through the cost of failure based on the MLH model.
\end{abstract}

\section{Introduction}

This paper aims at demonstrating the efficient application of Axiomatic Design (AD) on a case study, where AD has been applied to improve the reliability of a fire-fighting pump set. The need for this improvement can be traced back to the fact that, in a global market where goods are easily and more available to customers, quality and reliability play a fundamental role for companies competitiveness. Innovation has become one of the most important factors for the long-term success of a company, since increased customers expectations and growing international competition call for massive product variety. Companies have had to respond to customers demand to survive on the market, and they have had to be quick in developing new products due to the shortening of product life cycle [1]. In this scenario, the design process covers a fundamental role. Engineering design is usually divided into conceptual design and detailed design [2]. Conceptual design is carried out at the earliest stage of engineering design, and the most important decisions are formulated at this stage, including the overall functional requirements and characteristics of a product. Size and shapes of components are instead determined in the detailed design stage, using numerical analyses and experiments. On the one hand, wrong decisions taken in the conceptual stage are recognized to affect subsequent decisions and processes of the product, and can lead to a major defect in the product. On the other hand, wrong decisions in the detailed design stage can impact the reliability and the overall quality of the product. Failure in one of these stages can be very hard to cope with when the product is already in production; moreover, the iterative steps required to correct mistakes can be either very expensive or, in worst case scenarios, insufficient. Therefore, a rational and methodological approach is desirable to avoid issues caused by

\footnotetext{
^e-mail: p.placidoli@unimarconi.it
}

wrong decision-making during both the above-mentioned product development stages. The process of reliability improvement is in fact traditionally carried out at subsystem/component level in the last stage of design development. Classical techniques, characterized by the cycles of build-test-evaluate-fix, thus significantly increase the product development time and life cycle cost. As it has been established by Suh in [3], it is known that AD can efficiently avoid those issues, as it suggests a systematic approach which divides a large design problem into manageable small sub-problems that make the design process less prone to decision errors.

As per reliability itself, a simplification that is often done is the independence of component failure. Some studies on reliability in the automotive industry [4], among which the fundamental work on FMEA [5], have instead shown that only a small part of the system failure is caused by the failure of the single component. On the contrary, the most frequent cause is the interaction between subsystems or bad designed interfaces. The state of a system or a component is simplified in "success" or "failure" following the binary state assumption. Although binary approach can be appropriate for reliability goal setting, it gives few clues for engineering design regarding the improvement of design parameters [6]. The Multi-Level Hierarchical (MLH) method proposed by Hubka and Eder [7] and after enhanced by Trewn and Yang [8], integrating the reliability of functions with the concept of dependent failure and cost of failure, is able to overcome the above-mentioned critical issues.

In this study, the AD methodology and the MLH analytical system are applied together to redesign the supporting structure of a fire-fighting pump set produced by DAB Pumps Spa. During the design phase, two concepts (that we have indicated in the study as Alpha and Beta) have been evaluated to find the best design in terms of reliability, lower complexity, and expected cost of failure. 


\section{Methodology}

Theory of the resolution of invention-related tasks (TRIZ) [9], Failure Mode and Effect Tree Analysis (FMETA) [10], and Fault Tree Analysis (FTA) [11, 12] are among several methodologies that have been developed and applied to improve system reliability in various domains. TRIZ, for instance, has been used - among several applications to increase quality and reliability with regard to the manufacturing process of an industry that designs and builds molds and equipment to produce aluminum food containers [13]. FMETA looks for the most critical characteristics of the product from a reliability point of view, and provides the designer with a set of possible changes. FMETA has been successfully applied, for instance, to develop a reliability tree to evaluate both the RPN for the component of the product and to find the reliability relation useful for the following optimization, validated by an application to an automotive heavy-duty diesel engine [10]. FTA, namely, Comparative FTA, has proved useful to compare the reliability of an electronic braking system with its mechanical counterpart [14]. Complexity is defined by Suh [15] as the measure of uncertainty in satisfying functional requirements (FRs), caused by poor design or by lack of knowledge (or understanding) about the system under consideration. The versatility of AD has indeed been successfully tested in its application to several contexts, among which the authors have worked on virtual studios and television recording [16] within the product domain, and, within process domain, they have studied an application of AD to improve the process of airport assistance to patients with reduced mobility [17]. Due to its rate of successful applications, as already stated above, the novelty of this paper is the application of AD together with MLH, as the most suitable methodology to be applied to improve the reliability of the fire-fighting pump set. Therefore, a comparison has been established and debated among the best design solutions from the point of view of system components reliability. Ultimately, this paper aims at successfully applies AD integrated with MLH to optimize the design and improve the reliability of a machinery.

\subsection{Axiomatic Design Principles}

When applying $\mathrm{AD}$, the design activity can be schematized by using four domains: the customer domain, the functional domain, the physical domain and the process domain [3]. The customer domain describes the Customer Attributes (CAs); the functional domain (deducted from CAs) describes the design objectives and Functional Requirements (FRs); the physical domain provides Design Parameters (DPs) for the implementation of the FRs; the process domain translates the DPs into the Process Variables (PVs). Mapping among design domains is performed according to two axioms, representing the core of the methodology. The first axiom is called the Independence Axiom and it states that the independence of FRs must always be maintained. The second axiom is called the Information Axiom and it states that among those designs that satisfy the Independence Axiom, the best design is the one that has the smallest Information Content [3]. The AD mapping process between the functional domain and the physical domain is defined as follows:

$$
\{\mathrm{FR}\}=[A]\{\mathrm{DP}\}\left\{a_{i j}=(0,1)\right\}
$$

where $[\mathrm{A}]$ is the design matrix that maps the DPs to the FRs; $a_{i j}$ is the element of the matrix [A] that maps the $j$-th DP to the $i$-th FR. In this application, it is used as a design tool and the values of the matrix are assumed to be only 0 or 1 .

\subsection{Analytical Multi-Level Hierarchical Modeling}

As an independent development in the theory of Engineering Design, Hubka and Eder [7] defined a technical system at various levels of abstraction. It is called Multi-Level Hierarchical system and, similarly to AD, it describes the design process from the highest abstract level to the lowest concrete level. However, differently from AD, in MLH, after the physical space, the Component Space is also defined, describing the concrete Component Structure (CS) of the design. To merge AD and MLH, Trewn and Yang [8] developed a theoretical 2-step framework to characterize the relationship between functional reliability and component reliability with the presence of failure dependence. The underlying concept is that two case scenarios can occur in a process design:

- Several Design Parameters can be encapsulated into a single component.

- A single Design Parameter is delivered by using several components.

Therefore, the structure of relationships among components is often different than that of DPs. From a reliability perspective, it is the subsystem/component structure that determines the reliability, hence mapping the component reliability to the function structure determines the function reliability of the system. Basically, Trewn and Yang [2000] introduce the matrix [B] that maps the Design Parameters in the design space to the component structure in the component space.

Mathematically speaking:

$$
\{\mathrm{DP}\}=[\mathrm{B}]\{\mathrm{CS}\}\left\{b_{j k}=(0,1)\right\}
$$

Where $\{\mathrm{CS}\}$ is a vector with $\mathrm{k}$ components that characterize the component space and $b_{j k}$ is the element of the matrix [B] that maps the $k$-th component to the $j$-th design parameter. For the purpose of reliability analysis, the element $b_{j k}$ is defined to be binary, where $0=$ no relationship and $1=$ related. By combining the equations 1 and 2 :

$$
\{\mathrm{FR}\}=[\mathrm{D}]\{\mathrm{CS}\}
$$

where the matrix $[D]=[A] \circ[B]$ represents the relationship between the FRs and the components and it maps the relationship of each component of the system to the FRs that satisfies. The operator (o) is a composite relational operator for binary matrices. Finally, the reliability model is 
established by integrating failure probabilities of the components into this hierarchical multilevel model through the following equations:

$$
R_{s}=\prod_{i=1}^{m} P\left(\mathrm{FR}_{i}\right)
$$

where $P\left(F R_{i}\right)$ is the probability that $F R_{i}$ is successfully delivered and:

$$
P\left(\mathrm{FR}_{i}\right)=\prod_{k=1}^{n}\left(1-p_{k}\right)^{d_{i k}}
$$

where $d_{i k}$ is the entry of [D] matrix in $i$-th row and $k$-th column and $p_{k}$ is the failure probability of component $\mathrm{k}$. Clearly, $d_{i k}=1$ indicates that $k$-th component will affect $F R_{i}$ and vice versa. Hence:

$$
R_{s}=\prod_{i=1}^{m} \prod_{k=1}^{n}\left(1-p_{k}\right)^{d_{i k}}=\prod_{k=1}^{n}\left(1-p_{k}\right)^{\bigcup_{i=1}^{m} d_{i k}}
$$

This model allows to analyze the exact impact on the product of component failure, and to assess the damage of the failure with greater accuracy.

\subsection{Cost of Failure}

Within a system, when failure of a component occurs, it may cause a number of components to fail simultaneously. It is said that such failure is a dependent failure. In this case, these components may have to be repaired or replaced. However, the components usually affect the system in different ways. For example, they can affect either some minor functions of the product or some key functions that, in case of failure, can cause the entire system failure. Moreover, different components have different repair/replacement costs. In the MLH model discussed earlier, the following failure cost model is introduced to analyze the cost of component and function failures on system performance:

$$
E\left[\mathrm{C}_{\mathrm{CS}_{k}}\right]=\sum_{j=1}^{n}\left[p_{k \mid j} \times p_{j}\right]\left(\mathrm{CR}_{\mathrm{CS}_{k}}+\sum_{i=1}^{m} d_{i k} \mathrm{C}_{\mathrm{FR}_{i}}\right)
$$

Where:

$E\left[C_{C S_{k}}\right]=$ Expected cost of failure of component $\mathrm{k}$ $C R_{C S_{k}}=$ Cost of replacement of component $\mathrm{k}$ $C_{F R_{i}}=$ Cost of loss of function i

Equation 7 states that the expected cost of failure due to component $\mathrm{k}$ is proportional to the component replacement cost and the cost of losing relevant product functions affected by the failure of component $\mathrm{k}$.

\section{Case Study}

\subsection{Fire-Fighting Pump Set Description}

The fire-fighting pump sets are part of fire sprinkler system's water supply used in residential, public and commercial buildings, hospitals, and industrial plants. Firefighting pump sets are needed when the local municipal

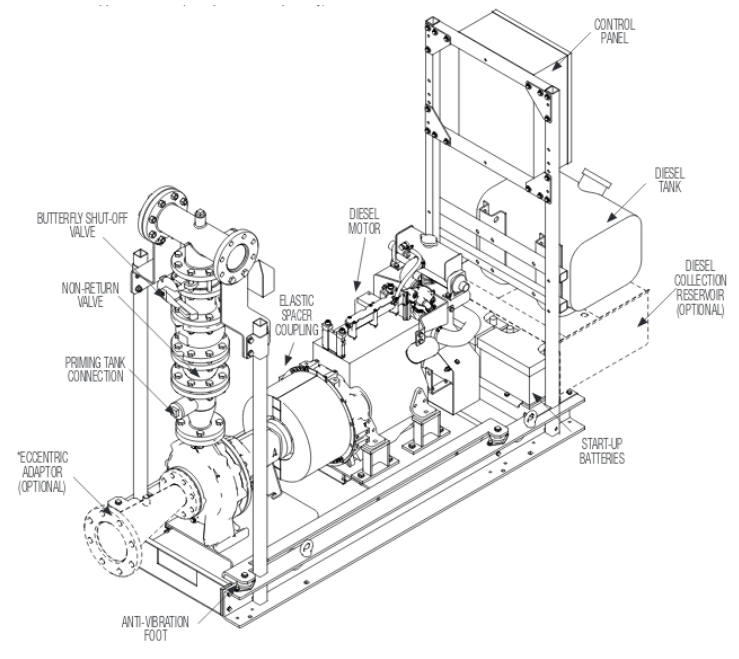

Fig. 1. Fire-fighting pump set

water system cannot provide sufficient pressure to meet the hydraulic design requirements of the fire sprinkler system prescribed by UNI EN 12845:2015. This usually occurs if the building is very tall, such as in high-rise buildings, or in systems that require a relatively high terminal pressure at the fire sprinkler in order to provide a large volume of water. The system is usually powered by diesel and electric motors, and typically connected to the public underground water supply piping, or a static water source (e.g., tank, reservoir, lake). The pump provides water flow at a higher pressure to the sprinkler system risers and hose standpipes. It starts and releases water when one or more fire sprinklers are exposed to a heat level which is beyond their design temperature, and the pressure in the fire sprinkler system drops below a certain set threshold. Alternatively, the fire-fighting pump set starts due to other fire hoses reels or other firefighting connections opening, causing a pressure drop in the fire fighting main.

\subsection{Fire-Fighting Pump Set Redesign Motivation}

After a market investigation that involved both partners and customers, the following needs were assessed:

- Reducing components cost to be more competitive on market.

- Increasing the overall reliability of the fire-fighting set to reduce the extraordinary maintenance costs met by the customers.

To accomplish these tasks, negotiations with dealers have brought to use different components (e.g. diesel motor, control panel) instead of the old ones. Such substitution of the components is the main reason for the redesign of the existing fire-fighting pump set shown in Figure 1. Due to the partial incompatibility between the newly chosen components and the supporting structure, the redesign process has focused on the developing of a new supporting frame entirely designed and built by DAB Pumps. 
Table 1. [A] Matrix

\begin{tabular}{lll}
\hline & $D P_{1}$ & $D P_{2}$ \\
\hline$F R_{1}$ & 1 & 0 \\
$F R_{2}$ & 0 & 1 \\
\hline
\end{tabular}

\subsection{Fire-Fighting Pump Supporting Frame Redesign}

Following the $\mathrm{AD}$ method, the following functional requirements and design parameters are chosen for the frame:

$F R_{1}=$ Support the fire-fighting pump set components

$F R_{2}=$ Limit the fire-fighting pump set vibration while operating

$$
\begin{aligned}
& D P_{1}=\text { Fixed frame } \\
& D P_{2}=\text { Anti-vibration system }
\end{aligned}
$$

The design matrix $[\mathrm{A}]$ is shown in Table 1. The mapping between the physical space and the component space defined by the MLH theory is performed by applying the equation 2. At this stage, the definition of components of the supporting frame (i.e the CS vector) has lead to two different design concepts called Alpha (Figure 2) and Beta (Figure 3), the CSs of which are:

\section{Alpha concept}

$C S_{1}=$ Anti-vibration rubber bells

$C S_{2}=$ Pump base

$C S_{3}=$ Anti-vibration foot abutment

$C S_{4}=$ Diesel motor base

$C S_{5}=$ Longitudinal beams

$C S_{6}=$ Control panel pillars

\section{Beta concept}

$C S_{1}=$ Anti-vibration rubber bells

$C S_{2}=$ Pump base

$C S_{3}=$ Bracket support of the uprights

$C S_{4}=$ Diesel motor base

$C S_{5}=$ Longitudinal beams

$C S_{6}=$ Control Panel pillars

The mapping between the DP and CS domain is completed by building the [B] matrix for each design concept, table 2 and 3 . These matrices are built considering the structural dependence between each DP and the chosen structural component. For example regards to the Alpha concept (table 2), $D P_{1}$ depends on $C S_{2,4,5,6}$, since they are the components that detail the fixed frame and hence carry out the function of supporting the fire-fighting pump

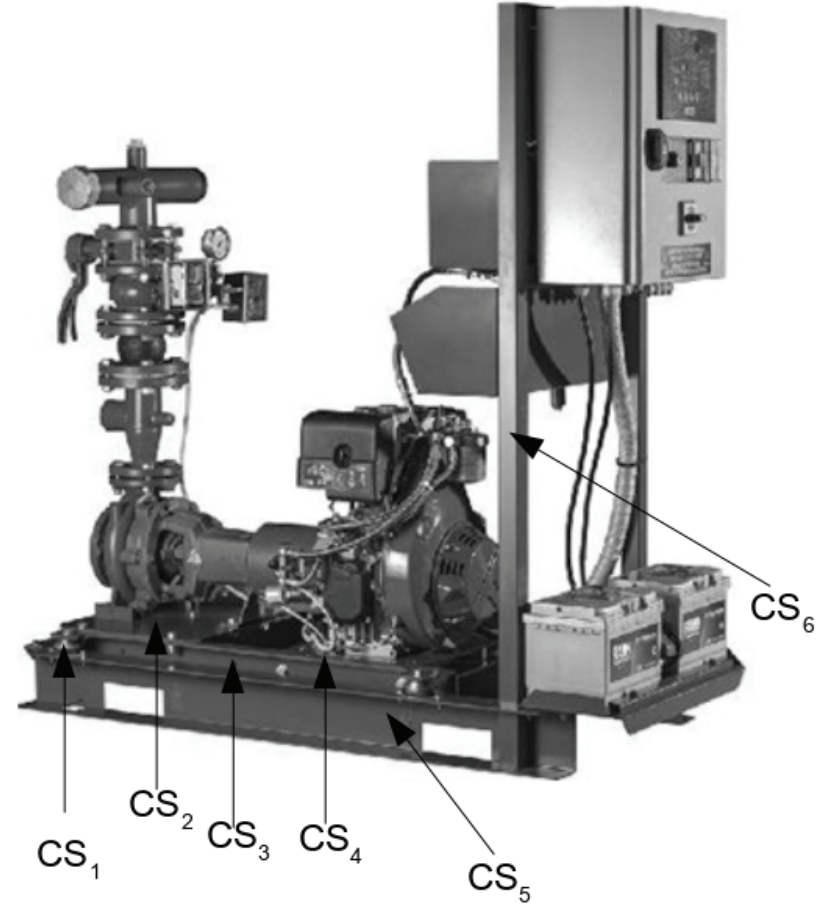

Fig. 2. Alpha design

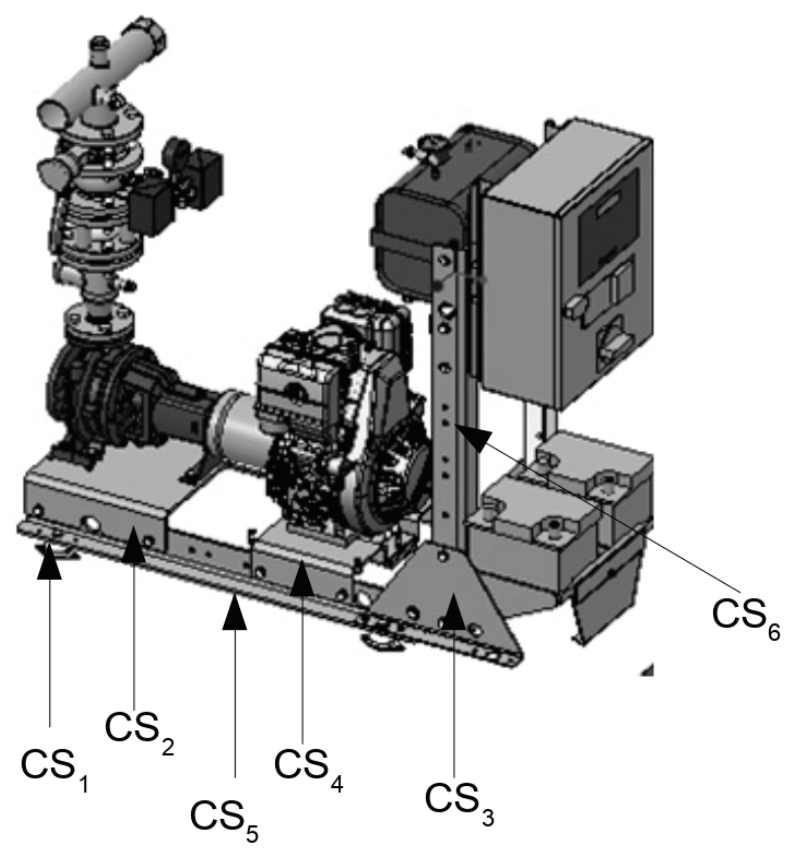

Fig. 3. Beta design

Table 2. [B] Matrix - Alpha

\begin{tabular}{lllllll}
\hline & $C S_{1}$ & $C S_{2}$ & $C S_{3}$ & $C S_{4}$ & $C S_{5}$ & $C S_{6}$ \\
\hline$D P_{1}$ & 0 & 1 & 0 & 1 & 1 & 1 \\
$D P_{2}$ & 1 & 0 & 1 & 0 & 1 & 0 \\
\hline
\end{tabular}


Table 3. [B] Matrix - Beta

\begin{tabular}{lllllll}
\hline & $C S_{1}$ & $C S_{2}$ & $C S_{3}$ & $C S_{4}$ & $C S_{5}$ & $C S_{6}$ \\
\hline$D P_{1}$ & 0 & 1 & 1 & 1 & 1 & 1 \\
$D P_{2}$ & 1 & 0 & 0 & 0 & 1 & 0 \\
\hline
\end{tabular}

Table 4. [D] Matrix - Alpha

\begin{tabular}{lllllll}
\hline & $C S_{1}$ & $C S_{2}$ & $C S_{3}$ & $C S_{4}$ & $C S_{5}$ & $C S_{6}$ \\
\hline$F R_{1}$ & 0 & 1 & 0 & 1 & 1 & 1 \\
$F R_{2}$ & 1 & 0 & 1 & 0 & 1 & 0 \\
\hline
\end{tabular}

Table 5. [D] Matrix - Beta

\begin{tabular}{lllllll}
\hline & $C S_{1}$ & $C S_{2}$ & $C S_{3}$ & $C S_{4}$ & $C S_{5}$ & $C S_{6}$ \\
\hline$F R_{1}$ & 0 & 1 & 1 & 1 & 1 & 1 \\
$F R_{2}$ & 1 & 0 & 0 & 0 & 1 & 0 \\
\hline
\end{tabular}

$\left(F R_{1}\right) . C S_{1,3,5}$ are instead the components chosen to detail $D P_{2}$ and to fulfill the anti-vibration function $\left(F R_{2}\right)$. The same metrics is used to build the [B] matrix for the Beta concept (table 3 ). In both cases $D P_{1}$ and $D P_{2}$ are coupled by the longitudinal beams $\left(C S_{5}\right)$ that are structurally connected with the anti-vibration rubber bells; hence, they can be considered part of both the fixed frame and anti-vibration system. The reliability of the functional requirements for both concepts is determined by equation 6 given the failure probability $p_{k}$ estimated for each component (table 6) and the related [D] matrix (Table 4 and 5):

\section{Alpha concept}

$$
\begin{aligned}
R_{F R_{1}}= & (1-0.05)^{0}(1-0.1)^{1}(1-0.01)^{0} \\
& (1-0.1)^{1}(1-0.1)^{1}(1-0.01)^{1}=0.7217 \\
R_{F R_{2}}= & (1-0.05)^{1}(1-0.1)^{0}(1-0.01)^{1} \\
& (1-0.1)^{0}(1-0.1)^{1}(1-0.01)^{0}=0.8465
\end{aligned}
$$

\section{Beta concept}

$$
\begin{aligned}
R_{F R_{1}}= & (1-0.3)^{0}(1-0.1)^{1}(1-0.1)^{1} \\
& (1-0.1)^{1}(1-0.01)^{1}(1-0.01)^{1}=0.7145 \\
R_{F R_{2}}= & (1-0.3)^{1}(1-0.1)^{0}(1-0.01)^{0} \\
& (1-0.1)^{0}(1-0.1)^{1}(1-0.01)^{0}=0.63
\end{aligned}
$$

Out of the comparison among the reliability values of the two designs, it is possible to highlight that the function with the worst reliability in the Alpha concept is the function of support the fire-fighting pump set components $\left(F R_{1}\right)$, while the function with the worst reliability in the Beta concept is the function of the supporting frame of limiting the vibration of the system $\left(F R_{2}\right)$. This poor reliability of $F R_{2}$ of the Beta concept is primarily due to the poor reliability of the anti-vibration rubber bells $\left(C S_{1}\right)$
Table 6. Failure probability (p) of components.

\begin{tabular}{lll}
\hline & Alpha & Beta \\
\hline$p_{1}$ & 0.05 & 0.3 \\
$p_{2}$ & 0.1 & 0.1 \\
$p_{3}$ & 0.01 & 0.01 \\
$p_{4}$ & 0.1 & 0.1 \\
$p_{5}$ & 0.1 & 0.1 \\
$p_{6}$ & 0.01 & 0.01 \\
\hline
\end{tabular}

that depends on the assembly of the Beta concept. The pump base, the motor base and the longitudinal beams are in fact positioned on the anti-vibration rubber bells that in this scenario become more subject to wear.

From a cost perspective, the cost of failure needs to be considered in order to select the best concept. To proceed with the calculation, the matrix $[\mathrm{P}]$ is built for each design, and it represents the dependence failure between components and, therefore, the possibility that a single failure mode may cause a number of components to fail simultaneously. In table 7 end 8 are represented the matrix entries with the cost of replacement $\left(C R_{C S_{k}}\right)$ of each component structure $\left(C S_{k}\right)$. Given the cost of loss of function for $F R_{1}=300 €$ and for $F R_{2}=100 €$, both of them consider the cost of the damage to the client, due to the failure of the system, and the cost related to the assistance service needed for the inspection and the repairing. Considering the scenario where failure occurs of the longitudinal beams $\left(\mathrm{CS}_{5}\right)$ of the Alpha concept, the calculation of the expected cost of failure applying the equation 7 is the following:

$$
\begin{gathered}
E\left[C_{C S_{5}}\right]=\left[\left(p_{5 \mid 1} p_{1}\right)+\left(p_{5 \mid 2} p_{2}\right)+\left(p_{5 \mid 3} p_{3}\right)+\right. \\
\left.+\left(p_{5 \mid 4} p_{4}\right)+\left(p_{5 \mid 5} p_{5}\right)+\left(p_{5 \mid 6} p_{6}\right)\right] \cdot \\
\cdot\left(C R_{C S_{5}}+\sum_{i=1}^{m} d_{i 5} C_{F R_{i}}\right)=[(1 \cdot 0.05)+(0 \cdot 0.1)+(0 \cdot 0.01)+ \\
+(0 \cdot 0.1)+(1 \cdot 0.1)+(1 \cdot 0.01)] \cdot[45+(1 \cdot 300+1 \cdot 100)]=71.2 €
\end{gathered}
$$

Clearly, the introduction of dependent failure analysis allows a broader and more thorough understanding about the damage caused by the failure of component 5. Assuming the independence, the expected cost of failure of component 5 is in fact $45.00 €$; however, by introducing the dependent failure model, it becomes $71.2 €$ due to the dependence of component 5 on the performance of components 1 and 6 . Once the procedure is applied for each component, the estimated cost of failure of the design is calculated by simply adding each $E\left[C_{C S_{k}}\right]$. The calculation for the two concepts, which is not shown here in view of a previous confidentiality agreement, has lead to select the Alpha concept as the one to have the best design, due to its less expected cost of failure.

\section{Conclusions}

The redesign of a supporting structure of a fire-fighting pump set has been addressed using AD and a MLH model 
Table 7. [P] Matrix - Alpha

\begin{tabular}{llllllll}
\hline$C R_{C S_{k}}$ & & $C S_{1}$ & $C S_{2}$ & $C S_{3}$ & $C S_{4}$ & $C S_{5}$ & $C S_{6}$ \\
\hline $10 €$ & $C S_{1}$ & 1 & 0 & 1 & 0 & 0 & 0 \\
$50 €$ & $C S_{2}$ & 0 & 1 & 1 & 1 & 0 & 0 \\
$42 €$ & $C S_{3}$ & 1 & 1 & 1 & 1 & 1 & 0 \\
$45 €$ & $C S_{4}$ & 0 & 1 & 1 & 1 & 0 & 0 \\
$45 €$ & $C S_{5}$ & 1 & 0 & 0 & 0 & 1 & 1 \\
$65 €$ & $C S_{6}$ & 0 & 0 & 0 & 0 & 1 & 1 \\
\hline
\end{tabular}

Table 8. [P] Matrix - Beta

\begin{tabular}{llllllll}
\hline$C R_{C S_{k}}$ & & $C S_{1}$ & $C S_{2}$ & $C S_{3}$ & $C S_{4}$ & $C S_{5}$ & $C S_{6}$ \\
\hline $10 €$ & $C S_{1}$ & 1 & 0 & 1 & 0 & 0 & 0 \\
$45 €$ & $C S_{2}$ & 0 & 1 & 1 & 1 & 0 & 0 \\
$55 €$ & $C S_{3}$ & 1 & 1 & 1 & 1 & 0 & 0 \\
$45 €$ & $C S_{4}$ & 0 & 0 & 1 & 1 & 0 & 0 \\
$35 €$ & $C S_{5}$ & 1 & 0 & 0 & 0 & 1 & 1 \\
$55 €$ & $C S_{6}$ & 0 & 0 & 0 & 0 & 1 & 1 \\
\hline
\end{tabular}

to evaluate the dependent failure of components and their related costs. The application of these procedures has allowed to carry the entire design process from the conceptual design stage to the detailed design stage. In particular, the MLH method has enabled the evaluation of two competing concepts in terms of uncertainty to functional delivery, thanks to the mapping process that has linked the functional requirements domain to the component structure domain. This systematic approach in conjunction with the dependent failure cost estimation has led to choose the best design with regard to lower complexity and improved reliability. The amount of information acquired during the design process has accelerated the decision-making process, while improving the entire design process, thus avoiding the trial-and-error approach typical of heuristic and empirical methodology.

\section{References}

[1] J. van Iwaarden, T. van der Wiele, The effects of increasing product variety and shortening product life cycles on the use of quality management system, International Journal of Quality \& Reliability Management 29, 470 (2012)

[2] D. Ullman, ed., The Mechanical Design Process (McGraw-Hill Education, New York, USA, 2015)

[3] N. Suh, ed., Axiomatic Design advances and applications (Oxford Univ. Press, New York, USA, 2001)
[4] G. Arcidiacono, J. Wang, Automotive Reliability, Quality and Reliability Engineering International 20, 3 (2004)

[5] P. Palady, ed., Failure Modes and Effects Analysis (PT Publication Inc, 1995)

[6] A. Giorgetti, C. Cavallini, G. Arcidiacono, P. Citti, A Mixed C-VIKOR Fuzzy Approach for Material Selection during Design Phase: A Case Study in Valve Seats for High Performance Engine, International Journal of Applied Engineering Research 12, 3117 (2017)

[7] V. Hubka, W.F. Eder, eds., Theory of Technical System (Springer-Verlag, 1988)

[8] J. Trewn, K. Yang, The relationship between system functions, reliability and dependent failures, in The 1988 IEEE International Conference on Systems, man and Cybernetics (San Diego, 1988)

[9] G. Altshuller, Creativity as an Exact Science: The Theory of the Solution of Inventive Problems (Gordon and Breach Science Publishers, New York, 1984)

[10] G. Arcidiacono, G. Campitelli, Reliability Improvement of a Diesel Engine using FMETA Approach, Quality and Reliability Engineering International Journal Special Issue on Automotive Reliability 20, 143 (2004)

[11] W. Schneeweiss, The fault tree method:(in the fields of reliability and safety technology) (LiLoLe-Verlag, 1999)

[12] Vesely, Goldberg, Roberts, Haasl, Tech. rep., Nuclear Regulatory Commission Washington dc (1981)

[13] G. Arcidiacono, L. Bucciarelli, TRIZ: Engineering Methodologies to Improve the Process Reliability, Quality and Reliability Engineering International Journal 32, 2537 (2016)

[14] G. Arcidiacono, Development of a FTA versus Parts Count Method Model: Comparative FTA, Quality and Reliability Engineering International Journal pp. 411-424 (2003)

[15] N. Suh, Complexity: theory and applications (Oxford University Press on Demand, 2005)

[16] G. Arcidiacono, P. Placidoli, Reality and illusion in Virtual Studios: Axiomatic Design applied to television recording, in Proceedings of ICAD2015, 9th International Conference on Axiomatic Design (Florence, Italy, 2015)

[17] G. Arcidiacono, A. Giorgetti, M. Pugliese, Axiomatic Design to improve PRM airport assistance, in Proceedings of ICAD2015, 9th International Conference on Axiomatic Design (Florence, Italy, 2015) 